\title{
Densification of powders by particle deformation
}

\author{
H. F. Fischmeister and E. Arzt
}

Based on a previous experimental study of particle deformation during powder compaction, a model is developed for describing the densification bchaviour of an irregular packing of spherical particles. Using the radial density function of a 'random dense packing', the increase in both the average size and the number of contact faces are calculated. A simple criterion for local yielding allows the compaction pressure to be determined for relative densities up to $90 \%$. In the final stage of compaction, particle deformation, now constrained by nejghbouring contacts, is modelled by extrusion into the remaining pore space. A compaction equation encompassing both stages is presented; its application to nonspherical powders elucidates the role of particle shape during powder densification. PM/0150

(C) 1983 The Metals Society. An earlior version of this paper was presented at the conference on 'Sintering: theory, praclice, development, and control held in London on 2224 October 1979; manuscript received in final form 28 July 1982. At the time the work was carried out the authors were at Montanuniversität Leoben, Austria; they are now with the Max-Plunck-Institut für Metallforschung, Sluttgarl, Federal Republic of Germany.

\section{LIST OF SYMBOLS}

a avernge contact area

$c$ slope of the RDF, according to $\operatorname{Scott}^{6} c=15.5$

$D$ relative density (volume fraction of the metal phase)

$D_{0}$ relative fill density

$f$ average contact force

$f_{h}, f_{s}$ volume fractions of 'hard' and 'solt' phase

$G(r)$ integrated radial densily function = average number of sphere centres (RDF) within radius $r$ from the centre of an arbitrary reference sphere

h coefficient of linear strain hardening

$H_{\mathrm{M}}(D)$ microhardness at contact flat, at density $\mathrm{D}$

$k$ geometrical constant

$L_{\mathrm{y}}$ length of triple lines/unit volume

$p$ compaction pressure

$P_{A}$ number of triple points (intersections of triple lines with plane of polish)/unit area of polished section

$R$ original particle radius

$R^{\prime}$ radius of splerical particles after fictitious growth (in units of the original particle radius $R$ )

$R^{\prime \prime}$ radius of the truncated spheres which are formed when the matcrial of overlap is uniformly distributed over the particle surface (in units of the original particle radius $R$ )

$s$ half the distance between the surfaces of the reference sphere and of a particle in its neighbourhood
$S_{0}$ surface portion on the reference sphere occupied by any initial neighbour $=2 \pi R^{\prime}\left(R^{\prime}-1\right)$

$S(s)$ surface portion on the reference sphere occupied by a sphere which, before compaction, was by $2 s$ too far to touch the reference sphere $=2 \pi R^{\prime}\left(R^{\prime}-1-s\right)$

$V_{0}\left(R^{\prime}\right)$ half the volume of overlap between a reference sphere and any initial neighbour $=\pi \cdot\left(R^{\prime}-1\right)^{2}\left(2 R^{\prime}+1\right) / 3$

$V(s)$ half the volume of overlap between a reference spherc and a sphere which, before compaction, was by $2 s$ too far to touch the reference sphere $=\pi \cdot\left(R^{\prime}-1-s\right)^{2}\left(2 R^{\prime}+1+s\right) / 3$

$Z$ average coordination number

$Z_{0}$ average coordination number before compaction

$\bar{\varepsilon}$ equivalent linear strain

$\sigma(\varepsilon)$ stress-strain relationship for a powder material

$\sigma_{\mathrm{r}}$ flow stress

$\sigma_{y}$ yield stress

Present descriptions of the pressure-density relationship during the compaction of metal powders appear inadequate because of oversimplification or the complete lack of a physical model. Obviously, the highly irregular shape of many industrial powders puts a general strict treatment of powder compaction beyond present reach. In recent years, however, spherical powders have come into commercial use in increasing quantities, and the compaction behaviour of such geometrically simple powders is not an unreasonable objective for theoretical modelling - at least if the treatment is restricted to spheres of a single size.

In trying to model compaction, it is helpful to distinguish three stages: during the first stage restacking (rearrangement, sliding) of particles occurs as discussed in a previous study. ${ }^{1}$ When this rearrangement is complete, a given particle has a certain number of contacting neighbours ('coordination number'). Almost from the beginning, and largely concurrently with restacking. particles which have already formed contacts will deform plastically. Progressive flattening brings the particle centres closer to each other, producing overall densification. During this process, further neighbours are brought close enough to form additional contacts - an effect which up to now has been largely neglected. This is the second stage of densification; it can be described by the model to be presented herc. Finally, as the compact approaches full density, its compressibility diminishes drastically (third
stage).

\section{Stage 1}

As has been shown previously, ${ }^{1}$ particle rearrangement is limited to the very beginning of compaction and does not contribute significantly to the densification of spherical powders. It is therefore omitted from the present theory.

\section{Stage 2}

At any moment during compaction the resistance of the powder to further densification will be determined by the 
number and size of the contact areas between the particles. A theoretical relationship between density and compaction pressure can be derived by dividing the problem into the following segments, which will be treated in turn:

\section{Geometry}

The density of the compact is determined by the amount of particle flattening, which can be characterized by the average contact area and the average coordination number. The connection between these quantities depends on the initial geometry of the particle packing.

\section{Plasticity}

The size of a given contact area depends on the local contact force and on the yield properties of the powder material.

\section{Force transmission}

The compaction pressure is propagated within the powder as a set of contact forces. In this model we use an expression for the average contact force as a function of external pressure.

\section{Stage 3}

Finally, neighbouring contacts on one particle will start to impinge, impeding the simple deformation mode which characterized the earlier stage of compaction. As compaction proceeds, more and more contacts will impinge, making an increasing fraction of the volume harder to compress. In the treatment proposed here, the magnitude of this volume is assessed by means of the "triple lines' which form where three particles join by mutual impingement of adjacent contacts. It will be demonstrated that in this final stage the densification behaviour is governed by the constrainls exerted by neighbouring contacts and that the asymptotic approach to theoretical density can be modelled with a formula from extrusion theory.

In summary, the gradually increasing resistance to densification will be explained quantitatively by the growth of contact areas, the creation and subsequent growth of additional contacts, the change of plastic properties of the powder material during compaction (strain hardening), and, finally, the increasing constraint under which particle deformation has to proceed.

\section{AVERAGE CONTACT AREA AND COORDINATION NUMBER $\nu$. DENSITY}

Experimentally it has been found that the number of particle contacts at a given sphere increases approximately linearly with the compaction pressure. ${ }^{\prime}$ The external pressure is therefore sustained by an increasing number of
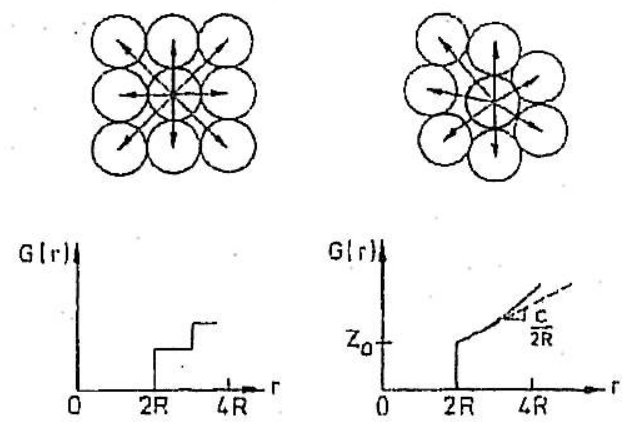

1 Integrated radial density functions (RDF) for (laft) regular and (right) random packing of equal spheres (schematically): RDF is number of sphere centres located within distance $r$ from reference sphere; in the narrow range of interest, RDF for a 'random dense packing' according to Scott' $^{5}$ can be approximated by a straight line; $A$ is particle radius particlc contacts and the resistance of the powder to further densification is raised more than would be cxpecled from a simple two-sphere model. This important feature of powder compaction is the consequence of the distribution of initial centre-to-centre particle distances. It cannot be modelled by assuming regular (lattice type) packings as in the models of Pelzel ${ }^{2}$ and Kakar and Chaklader; ${ }^{3,4}$ a continuously increasing coordination is conceivable only for an irregular particle structure.

A convenient way of characterizing an irregular packing is by means of the integrated 'radial density function' (RDF), which states the number of particle centres located within a sphere of radius $r$ uround an arbitrary reference sphere (Fig. 1). Scott ${ }^{5,6}$ has determined the RDF for a 'dense random packing' of equal spheres; since his packing has virtually the same coordination number and density as a bed of spherical powder particles before compaction, ${ }^{1}$ we shall use his result for the present theory. In the range of interest, Scott's $\mathrm{RDF}^{5}$ can be well approximated by

$$
G(r)=Z_{0}+c\left(\frac{r}{2 R}-1\right) \text {. . . . . . . . . . }
$$

$Z_{0}=7 \cdot 3$, which corresponds to the initial coordination number; $c=15 \cdot 5$; and $R$ is the particle radius.

Slarting from this RDF for the initial particle packing, we assume that during densification all centre-to-centre distances shrink by the same relative amount. Under these conditions, which could be termed "homogeneous densification', the shape of the RDF will remain unchanged.

The treatment of homogeneous densification is facilitated if, instead of letting the centre-to-centre distances shrink, we let the particles grow around their fixed centres, which results in a geometrically equivalent situation. A short calculation yields the new particle radius $R^{\prime}$ (in units of the original radius $R$ ) for a given relative density $D$

$$
R^{\prime}(D)=\sqrt[3]{\frac{D}{D_{0}}} \text {. . . . . . . . . . . . . }
$$

$D_{0}$ is the relative fill density (for monosize spheres $D_{0} \approx 0.64$, see $\left.S \cot t^{5}\right)$.

As a result of this sizc change, initially contacting spheres will now overlap (Fig. 2). In the powder, this corresponds to the squeezing out of material from the contact zones. For lack of an analytic plasticity treatment of the deformation problem, we shall distribute the excess material evenly over those parts of the sphcre surfaces that are not taken up by the contacts. This redistribution produces a further incrcase in the sphere radius from $R^{\prime}$ to $R^{\prime \prime}$, except at the contacts where the spheres are truncated.

The new particle radius can be calculated from a balance of volume

$$
V_{0}\left(R^{\prime}\right) Z_{0}+c \int_{s=0}^{R^{\prime}-1} V(s) \mathrm{d} s
$$

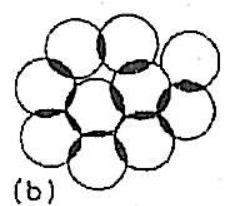

2 How the model works: in a packing of equal spheres (a) we let the particles grow around fixed centres until a certain density $D$ is reached (new particle radius $R^{\prime}(b)$; overlapping material is then deposited on free surfaces of spheres (now radius of 'truncated' spheres $\left.R^{\prime \prime}\right)(c)$; value of RDF for sphere centres at $r=2 A^{\prime}$ determines current coordination number, average contact area is function of $A^{\prime \prime}$ (oquation (5)) 
where

$$
\begin{aligned}
& V_{0}\left(R^{\prime}\right)=\frac{\pi}{3}\left(R^{\prime}-1\right)^{2} \quad \text { is half the volume of the overlap } \\
& \times\left(2 R^{\prime}+1\right) \text { between a reference sphere and } \\
& \text { any initial neighbour after } \\
& \text { growth from } R=1 \text { to } R^{\prime}
\end{aligned}
$$

The left hand side of equation (3) consists of the yolume of overlap between the reference sphere and its initial neighbours plus the contribution from newly formed contacts, which are created at a rate determined by the RDF of the packing. The right hand side of equation (3) states the volume of material which can be deposited on the sphere surface not taken up by contacts (initial and new). Equation (3) does not take into account the increase in coordination and the decrease of free surface area while the splieres are growing by addition of the excess material. These second order effects could be included by applying equation (3) iteratively. ${ }^{17}$ Since the present level of sophistication allows a stage 2 model to be built which works up to densitics of about $90 \%$ (when triple line formation sets in and a new mechanism becomes active, to be discussed below), the simplifications appear to be reasonable.

Integrating and solving equation (3) for $R^{\prime \prime}$ yield

$$
R^{\prime \prime}=R^{\prime}+\frac{4 Z_{0}\left(R^{\prime}-1\right)^{2}\left(2 R^{\prime}+1\right)+c\left(R^{\prime}-1\right)^{3}\left(3 R^{\prime}+1\right)}{12 R^{\prime}\left[4 R^{\prime}-2 Z_{0}\left(R^{\prime}-1\right)-c\left(R^{\prime}-1\right)^{2}\right]}
$$
The average contact area can be calculated as a function of
$R^{\prime \prime}$

$$
\frac{a\left(R^{\prime \prime}\right)}{R^{2}}=\frac{\pi}{3 Z R^{\prime 2}}\left[3\left(R^{\prime \prime 2}-1\right) Z_{0}+c+c R^{\prime \prime 2}\left(2 R^{\prime \prime}-3\right)\right]^{*}
$$

$Z$, the average coordination number, is equal to the number of spheres located within $r=2 R^{\prime}$ from the reference spherc, i.e. the value of the RDF at $r=2 R^{\prime}$

$$
Z\left(R^{\prime}\right)=G\left(2 R^{\prime}\right)=Z_{0}+c\left(R^{\prime}-1\right) . \quad . \quad . \quad . \quad . \quad .
$$

(Equation (G) is rather more realistic than taking the value of the $R D F$ at $r=2 R^{\prime \prime}$, as suggested by an incautious use

Ty Setting $Z_{0}=7.3$ and $c=15.5$, equation (5) can be approximaled
by

$$
\frac{u(D)}{R^{2}} \approx\left(D-D_{n}\right) \pi( \pm 4 \%) \text { for } D \leqslant 0.9
$$

which is close to the result of Kakar and Chaklader ${ }^{3}$

$$
\frac{a(D)}{R^{2}}=\frac{2}{3 D_{0}}\left(D-D_{0}\right) \pi
$$

for regular sphere packings. In the present model, there is an important additional increase in the resistanco to densification which results from the increase in coordination (equation (6)) and
flow stress (equation (9)).

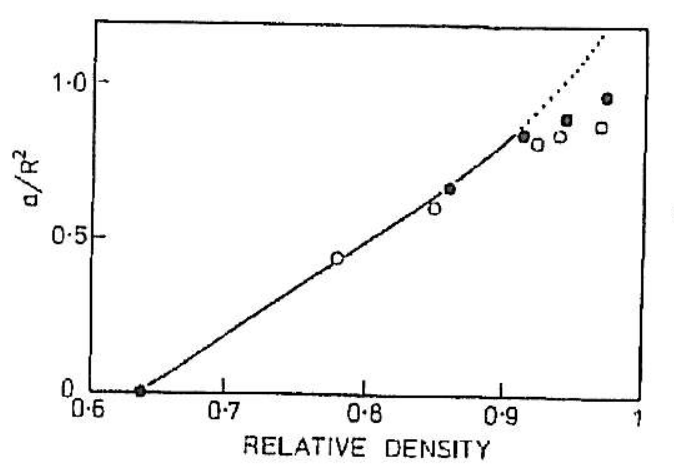

3 Average contact area between particles $v$, relative density of compact, obtained from measurements for spherical bronze powder' (o isostatic. O die compaction) and calculated according to equation (5)

of the model. During contact deformation in an actual powder, most of the material will stay in the vicinity of the contacts, and the formation of new contacts will therefore be due mainly to the shrinkage which corresponds to the sphere growth from $R$ to $R^{\prime}$ )

Thus wc have arrived at complete expressions relating the average contact arca and the average coordinution number to the relative density. Agreement with experimental results obtained in our earlier study for a spherical bronze powder ${ }^{1}$ is close up to a density of about 90\% (Figs. 3 and 4). Beyond this density level, the impingement of adjacent contacts (as discussed below) introduces a new deformation mode in the model. Therefore the overestimation of the contact area above $D=0.9$ (dotted line) is of no consequence. The coordination numbers predicted by cquation (6) ure low at high densitics because of the limitations of equation (3) mentioned above; again, the discrepancy concerns only the region where contact impingement will necessitale a new model in any case,

As will be seen below, it is the product $a Z$ which determines the resistance of the compact to further densification. Equation (5) happens to predict $a Z$ with good accuracy throughout the whole course of compaction (Fig. 5), although at high densities this appears to be fortuitous.

\section{CONTACT AREA p. CONTACT FORCE}

When two spherical particles are pressed against each other, the deformation is at first elastic. According to the Von Mises criterion the material should begin to yield when the mean pressure on the contact area l'eaches a value of about $1.1 \times$ the yield stress $\sigma_{y}$ of the material, ${ }^{7} \mathrm{Al}$ this stage the region of plasticity is rather small, but as the contact force is further raised, the plastic domain soon

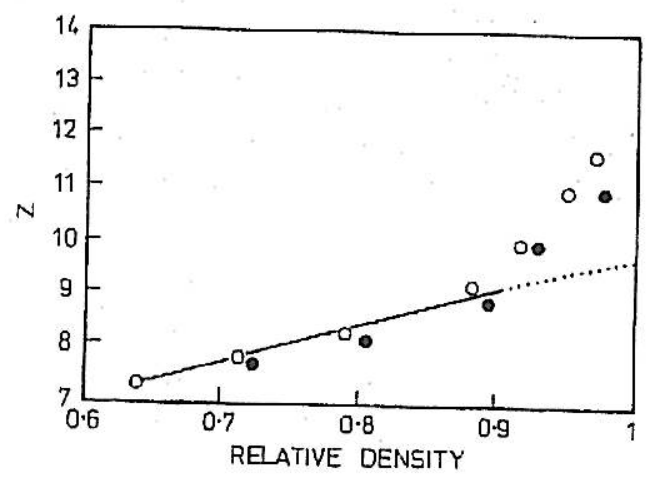

4 Average coordination number $v$. relative density, bronze powder' (o isostatic, O die compaction) and calculated according to equation (6) 


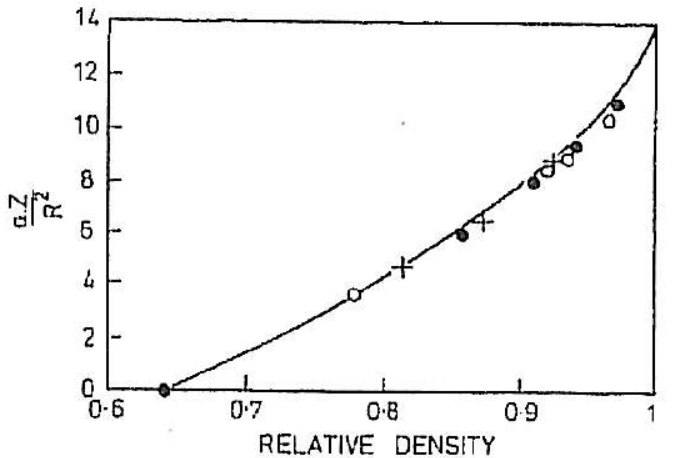

5 Product average coordination number $\times$ average contact area calculated from equation (5) compared with measured values' (o compacts from sieved. nearly monosize powder, + unsieved powder with wider size distribution)

extends over the whole contnct area.

The continuum-mechanical treatment of this deformation problem, despite its axial symmetry, presents serious difficulties which have not yet been resolved. Prandt ${ }^{\theta}$ has calculated the slip-line field for the basically similar (but two-dimensional) case of a frictionless cylindrical punch penetrating the flat face of an ideally plastic solid. He has found the onset of plastic flow to occur at a mein pressure of about $2.97 \sigma_{y}$. Similar analyses due to Hencky ${ }^{9}$ and Ishlinsky ${ }^{10}$ arrive at essentially the same result. Therefore, it seems reasonable to adopt as a pragmatic criterion for yielding (see also Kakar and Chaklader ${ }^{4}$ )

$$
f=3 \sigma_{y} a \text {. }
$$

where $f$ is the contact force.

This relation was verified by indentation experiments by Tabor, ${ }^{7}$ who found, however, that even in materials incapable of work hardening the numerical factor increased slightly with the size of the indentation. This was ascribed to the increasing constraints exerted by the displaced material.

In a packing of simultaneously deforming particles there will be even more pronounced constraints from neighbouring contacts. Sundström and Fischmeister ${ }^{1 !}$ have shown that such a 'geometrical hardening' is an inescapable consequence of the way in which a particle compact deforms, and that in the final stages of compaction it will play a role at least as important as the strain hardening of the particle material.

\section{STRAIN HARDENING OF THE POWDER} MATERIAL

Tabor ${ }^{7}$ further obscrved that a linear relation as in

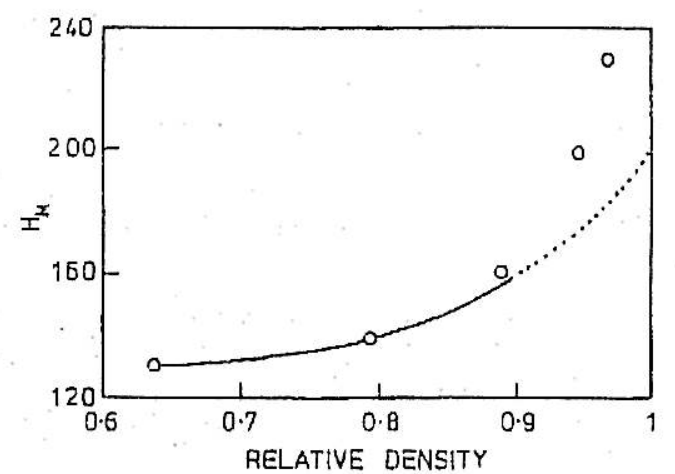

6 Microhardness at contact flats $v$. relative density: bronze powder, die compacted $(O)$ and calculated from equations $(8)$ and $(10)$

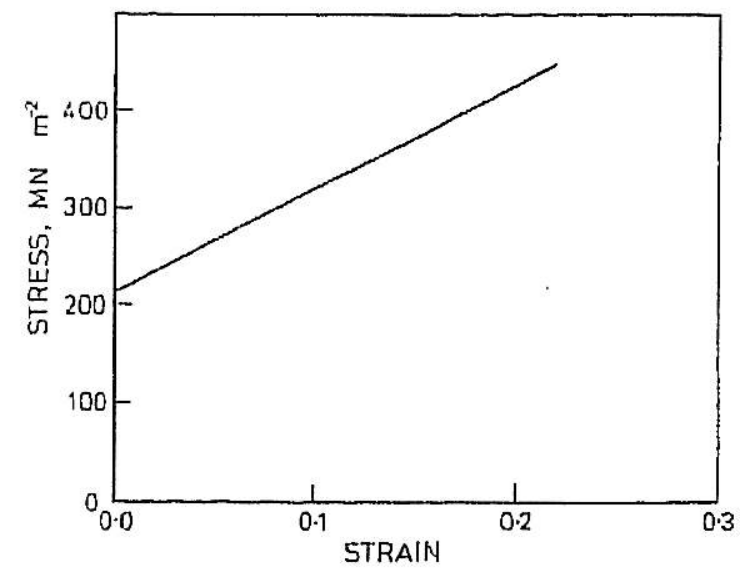

7 Stress-strain characteristic of fully dense bronze compacts (after Strömgren ${ }^{17}$ ) and linear fit used in model: $\sigma_{y}=200 \mathrm{MN} \mathrm{m}^{-2}$, linear strain hardening coefficient $h=6$

equation (7) holds also for work hardening mnterials if the flow stress of the deformed material at the edge of the indentation is inserted.

In the bronze powder compacts studied earlier, microhardness measurements werc performed in the centre of the contact flats to assess the amount of strain hardening that had taken place up to different stages of compaction (Fig. 6). Similar tesults have been reported by James ${ }^{12}$ for a variety of different materials,

For the bronze powder the stress-strain characteristic $\sigma(\varepsilon)$ had been determined from incremental compression tests performed on forged, fully dense specimens ${ }^{13}$ (Fig. 7). The equivalent strain $\bar{\varepsilon}$ in the deformed sphere can be assessed by viewing the fictitious growth of the spheres from $R=R^{\prime}$ to $R=R^{\prime \prime}$ as a plastic deformation process

$$
\bar{\varepsilon}=\ln \frac{R^{\prime \prime}}{R^{\prime}} \approx \frac{R^{\prime \prime}-R^{\prime}}{R^{\prime}} . \text {. . . . . . . . . . . }
$$

The flow stress $\sigma_{\mathrm{r}}$ is obtained by inserting this strain in the stress-strain relation $\sigma(g)$ of the particle material, which for the bronze powder (Fig. 7) can be fitted by

$$
\sigma_{\mathrm{r}}(D)=\sigma(\vec{\varepsilon})=\sigma_{y}(1+h \bar{\varepsilon})
$$

where $\sigma_{y}=200 \mathrm{MN} \mathrm{m}^{-2}$ and $h=6$. To test the validity of the strain assessment (equation (8)) the expected microhardness was computed according to

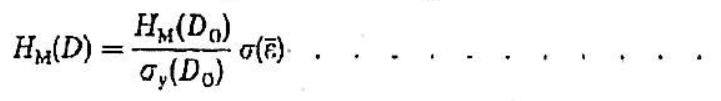

and compared with experimental results (Fig. 6). The agreement is good in the density range $(D<0.9)$ for which the model is intended.

\section{COMPACTION PRESSURE $v$. AVERAGE CONTACT FORCE}

The compaction pressurc is propagated in a powder as a set of forces acting at the points of contact between the particles. Considering the large number of particles and the randomness of the packing, it seems permissible to replace the actual force system by equal forces corresponding to the statistical mean. Then the following relationship can be derived $^{14}$

$$
f=\frac{4 \pi R^{2}}{Z D} P
$$

Equation (11) evokes a simple interpretation: the average contact force is the compaction pressure $x$ the average contact area, which is approximated by the sphere surface divided by $Z D$. A similar equation has been proposed by Coble and Ellis. ${ }^{15}$ 


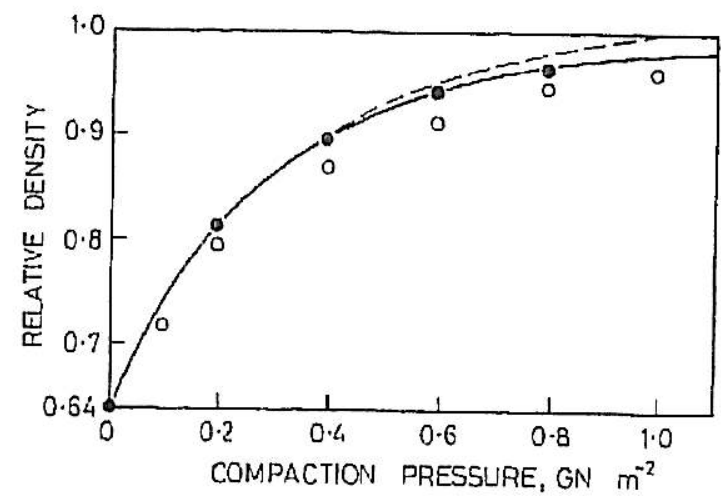

8 Experimental compaction data for spherical bronze powder ( $O$ isostatic, $O$ die compacted) and curves calculated according to equation (12). model of unconstrained deformation $(---)$ and equation (18). final model including constraint hardening $(\longrightarrow)$

\section{MODEL OF UNCONSTRAINED DEFORMATION (STAGE 2)}

Now we have all the elements for a first model of powder compaction, which will be called 'model of unconstrained deformation', because the deformation of each contact is assumed to occur independently of and without constraint from neighbouring contacts. It will break down at densities close to full density.

The pressure required to further densify a compact which has achieved relative density $D$, average contact area $a$, average coordination number $Z$, and flow stress $\sigma_{f}$ can be calculated by substituting equation (7) in to equation (11)

$$
p(D)=\frac{3}{4 \pi} \frac{a(D) Z(D)}{R^{2}} D \sigma_{\Gamma}(D) \text {. . . . . . . . . }
$$

All the terms involved have already been expressed as functions of relative density.

In Fig. 8 the compaction dala for the spherical bronze powder are compared with calculations according to cquation (12). The agreement is excellent throughout the technical pressure range. It is only in the very last stage of densification (stage 3) that the extremely strong increase in deformation resistance remains to be explained. Obviously normal strain hardening (for which a reasonable strength incrcase would be no more than twofold) cannot account

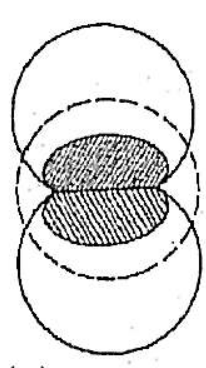

(a) (b)

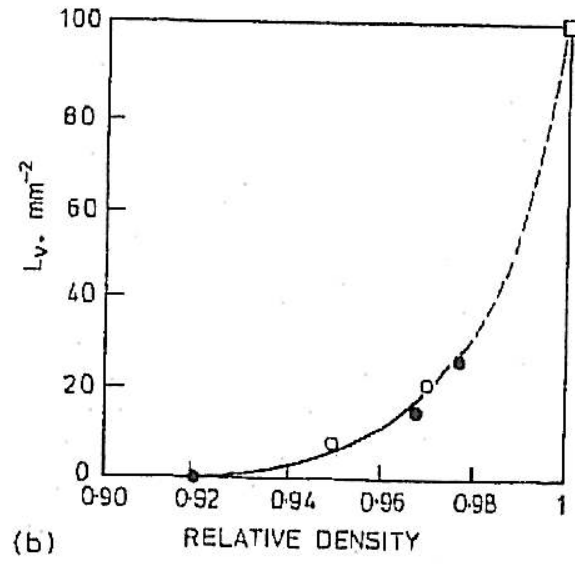

9 a formation of triple line in spherical powder: contact areas impinge on sphere surface (third sphere transparent for better illustrations): and $b$ length of triple lines/unit volume $v$. relative density (o isostatic, $D$ die compacted, $\square$ specific edge length of stack of tetrakaidecahedra, particie radius $R=143 \mu \mathrm{m}$ for the fact that the compact will become incompressible at full density. A first explanatory indication is provided by Sundström and Fischmeister, ${ }^{11}$ who emphasize the importance of 'geometrical hardening. In the following model for the final stage of densification this concept is developed further.

\section{GEOMETRICAL CONSTRAINTS AND} CONSTRAINT HARDENING (STAGE 3)

The growth of contact areas and the increase of the coordination number during densification will, at a certain stage, bring neighbouring contact flats on a given sphere surface close cnough to form a triple line (Fig. 9a). On a metallographic section the length $L_{\mathrm{V}}$ of the triple lines/unit volume can bc determined by counting the triple points (intersections of triple lines with the plane of polish)/unit area $^{16}$

$$
L_{\mathrm{v}}=2 P_{\mathrm{A}} \text {. . . . . . . . . . . . . . }
$$

where $P_{\mathrm{A}}$ is the number of triple points/unit area.

The increase of $L_{\mathrm{V}}$ in the bronze powder compacts is shown in Fig. 9b. In terms of the model, the formation of the first triple lines is to be expected as soon as the spheres have grown to a size which produces triple overlaps in a 'close' arrangement of three spheres, i.c. at $R^{\prime \prime}=\frac{2}{3} \sqrt{3}$. This corresponds to a relative density of about $90 \%$ which is not only in good agreement with measured triple line length (Fig. $9 b$ ), but also coincides with the density level at which the actual behaviour of the compacts starts to deviate from calculations based on unimpeded deformation.

Triple lines impede the further growth of contact areas, These constraints may raise the deformation resistance far beyond that expected from the model of unconstrained deformation. It is suggested that this last stage of compaction be termed the stage of 'constraint hardening' (where 'hardening' is used in its literal sense, i.e. to describe the increase in deformation resistance of the porous body as a whole).

A criterion for yielding under these constraints can be borrowed from the theory of extrusion ${ }^{18}$ and adapted to the present geometry

$$
p(D)=k \sigma_{\mathrm{f}} \ln \frac{a(1) Z(1)}{a(1) Z(1)-a(D) Z(D)} . . . \quad . \quad . .
$$

Here the logarithmic extrusion tatio has been expressed in terms of the contact area and the coordination number at density $D$ and at full density $D=1, k$ is a geometrical constant; $k=2$ leads to good agreement between theory and experiment.

\section{COMPLETE COMPACTION FORMULA}

Throughout compaction, the compact can be considered to consist of two kinds of regions; zones where the material is 'hard' (owing to triple lines) and regions where the deformation is still unconstruined. The volume fractions of these regions change conversely during compaction.

In order to combine both deformation mechanisms, we employ a linear mixture rule whose validity is well substantiated. ${ }^{19}$ It states that the resistance to deformation of a two-component material may be expressed as a linear combination of the properties of the components

$$
p=f_{\mathrm{h}} p_{\mathrm{h}}+f_{\mathrm{g}} p_{\mathrm{s}} \text {. }
$$

where $f_{\mathrm{h}}$ and $f_{\mathrm{s}}$ in this context denote the volume fractions of the 'harder' and 'softer' component, and $p_{\mathrm{h}}$ and $p_{\mathrm{s}}$ mean the yield criteria (equations (14) and (12)) for construined and unconstrained deformation, respectively. The volume fraction of the harder component can be assessed by the specific length $L_{\mathrm{v}}$ of the triple lines

$$
f_{\mathrm{h}}(D)=\frac{L_{\mathrm{V}}(D)}{L_{\mathrm{V}}(1)}
$$


and

$$
f_{5}(D)=1-f_{h}(D) \text {. }
$$

where $L_{v}(1)$ is the value of $L_{v}$ extrapolated to full density. The choice of numerical value is not very critical. If, according to our previous study, ${ }^{1}$ a stack of tetraknidecahedra (truncated octahedra) with edge length $L$ is used to approximate the fully dense compact, then $L_{\mathrm{V}}(1)$ is given by

$$
L_{\mathrm{v}}(1)=\frac{3}{2 \sqrt{2}} L^{-2} \approx 2.06 R^{-2}
$$

Combining equations (12) and (15)-(17) leads to the final compaction equation

$$
\begin{aligned}
p(D)= & \sigma_{y}(D)\left[\frac{3}{4 \pi} \frac{a(D) Z(D)}{R^{2}} D\left[1-f_{\mathrm{h}}(D)\right]\right. \\
& \left.+2 f_{\mathrm{h}}(D) \ln \frac{a(1) Z(1)}{a(1) Z(1)-a(D) Z(D)}\right] .
\end{aligned}
$$

The algebraic structure of this equation reflects the contributions of the two densification mechanisms. From the beginning of compaction up to a relative density of about $90 \%$, triple lines do not form, $f_{4}=0$, and the second term in parentheses remains without importance. After the first triple lines evolve, the extrusion term rapidly gains importance until finally it takes complete control of the densification process. The final predominance of the second term accounts for the asymptotic character of the approach to full density.

For the practical application of equation (18), the following approximate expressions are useful

$$
\begin{aligned}
& a(D) Z(D)=R^{2} \frac{D-D_{0}}{D}\left[160\left(D-D_{0}\right)^{2}+16\right] . . \\
& f_{\mathrm{h}}(D)=1953(D-0.92)^{3} \\
& \varepsilon=22 \cdot 2\left(\sqrt[3]{D / D_{0}}-1\right)^{3} \text {. . . . . . . . . . . . . . . . . }
\end{aligned}
$$

As can be seen in Fig, 8, equation (18) is in close agreement with measurements on isostatically compacted bronze particles (solid symbols). Less perfect agreement is to be expected for axial compaction in a die (open symbols) where the pressure is reduced by friction.

\section{DISCUSSION}

In equation (18), the characteristics of the flow and strain hardening behaviour of the particle material are collected in the first term. The second term is purely geometrical. It contains the initial density, the initial coordination number, and the slope of the RDF of the particle packing. In the case of monosize spheres, these quantities are all known and cannot be adjusted to fit the model to measurements. This is important because, in view of the smooth and simple shape of all compaction curves, the fact that a model equation can be fitted to expcrimental values by adjusting free parameters is almost worthless as verification of the model. Conversely, the good fit provided by equation (18) without adjustable parameters gives strong support to the conceptual basis of the model proposed in the present paper.

However, the model as conceived is limited to monosize spheres and it appears difficult to develop an equally stringent treatment for powders of varying particle size, and especially of irregular shape. From a pragmatic point of view, one might hope that the effects of not-too-large deviations in size und shape might 'average out' and try to apply the compaction function derived for monosize spheres also to less restricted powders.

Distributions of size and shape would affect the parameters $D_{0}, Z_{0}$, and $c$. Test calculations in which these quantities were varied one at a time showed that the shape

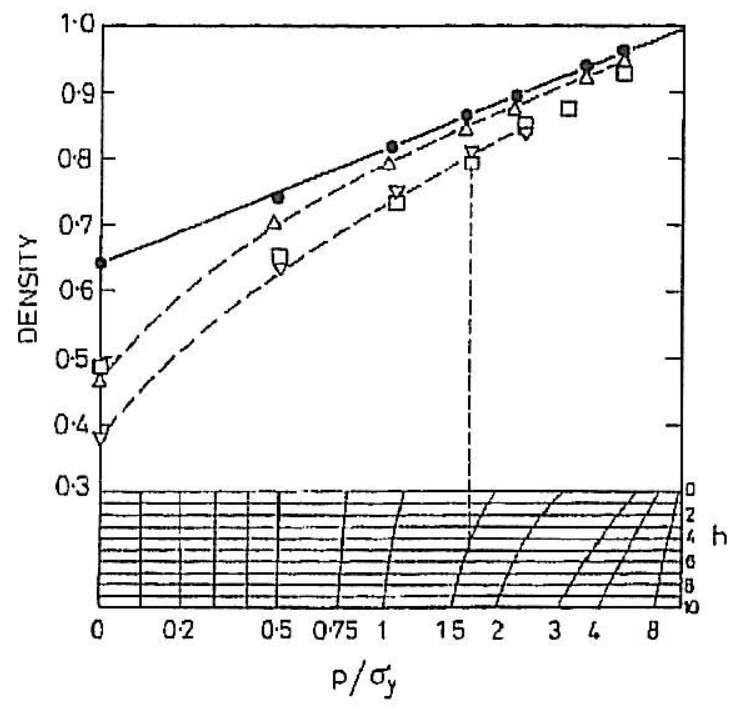

10 Compaction data for o bronze powder, spherical, after Fischmeister ot al.' $\triangle$ Atomet 28 iron powder, irregular, after Hewitt ot al, ${ }^{20}, \square$ aluminium, irregular, after Hewitt ot al..21, $\nabla$ stainless steel, irregular, after James, ${ }^{12}$ plotted in coordinates designed to give straight lines for powders which densify according to model: abscissa is shown for different rates $h$ of linear strain hardening

of the compaction curve is not very sensitive to $c$. Newly formed contacts have small areas and will not contribute much to the deformation resistance unless they appear in very large numbers. Noting that the decisive quantity in equation (18) is the product $a Z$, the idea suggests itself that an increase in $Z$ might often be offset by a decrease in $a$. Measurements were made of $a Z$ on compacts of bronze powvder with a size distribution from 100 to $350 \mu \mathrm{m}$. The results (shown as crosses in Fig. 5) are indeed found to coincide with those described carlier, which had been obtained with a narrow size fraction of the same powder.

The monosize sphere model allows only one initial density, that of the random dense sphere packing $D_{0}=0.64$. If $D_{0}$ is allowed to vary, it strongly affects the course of the compaction curve because it enters the compaction equation via equation (2). In fixing $D_{n}$ at 0.64 , the present treatment negates particle rearrangement before contact flattening. As has been shown in a previous study, ${ }^{1}$ this is not a serious simplification in the case of a spherical powder of a ductile material, but it would become so for irregular powders.

Plotting the compaction curves of various powders as reported in the literature in a net of curves generated from equation (18) by variation of $D_{0}$ showed that in the range of intermediate pressures, where particle rearrangement should have come to an end, most powders conformed well with the course of these calculated curves. For the regions of fit, onc could determine an effective value of $D_{0}$ which would signify the density at the moment from which densification proceeds by contact deformation alone. This partial coincidence with the sphere model might of course be fortuitous. Two observations suggest that it is not: first, the values of 'effective $D_{0}$ ' all fell into a narrow range, close to but never above the theoretical value of 0.64 ; second, the initial deviation (before confluence with the model curve) was found to correlate quite clearly with the irregularity of particle shape. This suggests that differences due to particle shape and sizc distribution become less and less important as the powder particles are squeezed logether, making the compaction behaviour of widely different powders converge towards that of spheres with progressively 

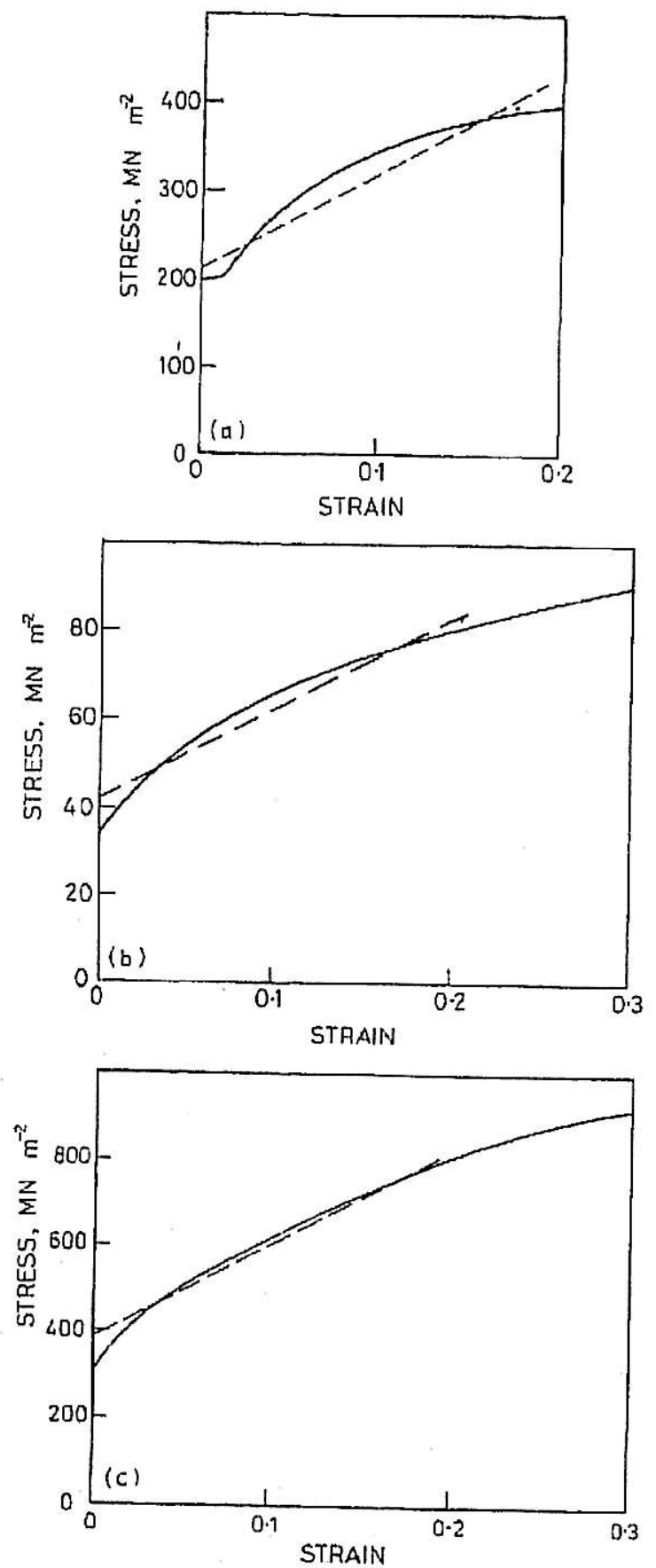

11 Stress-strain characteristics for a sintered Atomet 28 iron $_{2}^{20} \sigma_{Y}=200 \mathrm{MN} \mathrm{m}^{-2}, h=6 ; b$ aluminium, ${ }^{21}$ $\sigma_{y}=40 \mathrm{MN} \mathrm{m}^{-2}, h=6 ; c$ stainless steel, ${ }^{ \pm} \sigma_{y}=400$ MN $m^{-2}, h=5$; linear fits used for plotting data in Fig. 10

deforming contacts. If this is true, it should be revealing to plot compaction curves in such a way that the deviation from 'sphere behaviour' as well as the ultimate conformance with this behaviour could be seen and interpreted. A graph paper was constructed by graphical inversion of equation (18), on which contact flattening would produce as straight line (Fig. 10). Different values of $D_{0}$ will produce only parallel shifts of the line. In order to accommodate differences in the plastic flow behaviour of the particle material, the abscissa is graded for compaction pressure normalized by yield stress. A linear strain hardening law is assumed in the manner of equation (9). A grid of curves at the bottom of Fig. 10 allows the yield stress normalized pressure to be transformed so as to take care of strain hardening. The practical procedure is indicated by a sample trace in Fig. 10: for a material with a strain hardening rate $h=5$, a pressure of $1.5 \times$ the yield stress would be entered vertically above the point where the horizontal for $h=S$ is intersected by the grid curve corresponding to $p / \sigma_{y}=1.5$.

Figure 10 shows the behaviour of our spherical bronze powder together. with three other powders of different particle shape, for which data on compaction as well as strain hardening could be found in the literature. Figure 11 shows the degree of approximation involved in the assumption of linear strain hardening.

We believe that compaction curves plotted in the way of Fig. 10 (which expands their initial portions) reveal the rearrangement behaviour of the powder and the degree to which particle geometry hinders densification by contact flattening. The particle shape of the Atomet iron powder allows it to approach 'spherelike' behaviour when the relatively fine rugosity of the particle surfaces has been squeczed flat. The more irregular shapes of the aluminlum and stainless steel particles lead to more persistent deviations from the compressibility which would have been achieved with a more equiaxial structure. If verified, this interpretation of the difference in compaction behaviour would suggest ways of improving compressibility without too much sacrifice of green strength, and plots in the manner of Fig. 10 would give a quantitative indication of the scope for improvement by particle shape control. By analysing a large number of powders along these lines, one could develop more differentiated interpretations of the deviation of various parts of the compaction curyes from spherelike behaviour.

While these ideas are still hypothetical, we feel that they illustrate the type of practical conclusions which could be drawn from a well developed physical model for the densification of powders.

\section{ACKNOWLEDGMENT}

This project was supported by the Austrian Science Research Council (Fonds zur Förderung der wissenschaftlichen Forschung, Projekt 3181).

\section{REFERENCES} 1. H. ז. FISCHMEISTER, E. ARzT, and L. R. OLSSON: Poivder Metoll.,
$1978,21,179$.

2. E. PElzel: Z. Metallkd., 1955, 46, 813.

3. A. K. Kakar and A. C. D. ChaKLADER: J. Appl. Phys. 1967, 38, 3223.

4. A. K. KAKAR and A. C. D. CHAKLADER: Trans. AIME 1968, 242, 1117.

5. G. D. SCotT: Nature, 1962, 194, 956.

6. G. D. SCOTT: Nature, 1960, 188, 908.

7. D. TABOR: Proc. R. SOC, 1948, A192, 247.

8. L. PRANDTL: 'Úber die Härte plastischer Körper', 74; 1920, Göllingen, Nachr. Königl. Ges. d. Wiss.

9. H. HENCKY: Z. angew. Math. Mech., 1923, 3, 241 .

10. J. tshlunsky: J. Appl. Math. Mech. USSR, 1944, 8, 233.

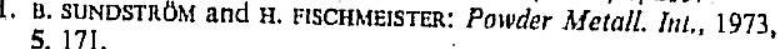
$5,17 \mathrm{I}$.

12. P. J. JAMES: Powder Metall., 1977, 20, 199.

13. M. STRÖMGREN: Diploma thesis, Chalmers Tckniskn Höyskola, Götebarg, 1974.

14. O. Molenus: Powder Technol., 1975, 12, 259.

15. R. L. COBLe and J. S. ellis: J. Am. Ceram. Soc., 1963, 46, 438.

16. C. S. sMitri and L. GUTTMAN: Trans. AIAE, 1953, 197, 81 .

17. E. ARzi: Acta Metall., 1982, 30, 1883.

18. F. A. MeCliNTOCK and A. S. AROON (ed.): 'Mechanical behaviour of materials', 367; 1966, Reading, Mass., Addison-
Wesley.

19. H. FUSCHMIEISTER and B. KARLSSON: Z. Metallkd., 1977, 68, 311.

20. R. L. HEWITT, W. WALlace and M. C. MALHERBE: Powder Metall., 1973, 16, 88

21. R. L. HeWITT, W. WALLACE and M. C. MALhereg: Int. J, Powder Metall. Powder Technol., 1974, 10, 131.

22. Data for Stainless Steel 310, Fagersta AB, Sweden. 